\title{
Doença de Still do adulto associada à hipertensão arterial pulmonar: relato de caso
}

\author{
Adult-onset Still's disease associated with pulmonary arterial hypertension: case report

\section{Enfermidad de Still del adulto asociada a hipertensión arterial pulmonar: reporte de caso}

Artênio José Saliba Garbin1, Rodrigo Borges Oliveira Cano ${ }^{1}$, Tony Maronesi Bagio ${ }^{1}$, Vinícius Cortellazze Lima ${ }^{1}$, José Marques Filho ${ }^{1 *}$.

\section{RESUMO}

Objetivo: Relatar a coexistência entre Doença de Still do Adulto e Hipertensão Arterial Pulmonar. Detalhamento do caso: Apresentamos o caso de uma gestante de 29 anos, encaminhada pelo serviço de reumatologia de um hospital do estado de São Paulo com diagnóstico de Doença de Still do adulto. Apresentou no início da doença febre persistente, artralgias, artrite bilateral em punhos, pericardite, derrame pleural, ferritina elevada, linfadenopatia e visceromegalia. Foi realizado parto cesárea e, dois meses depois, utilizou doses progressivas de prednisolona, após recidiva da doença. Apresentou evolução do quadro cursando com os mesmos sintomas, porém associada à hipertensão pulmonar ao exame ecocardiográfico. Transferida à Universidade de São Paulo para cateterismo cardíaco. Durante o exame apresentou parada cardiorrespiratória, sem resposta as manobras de ressuscitação. Considerações finais: A Doença de Still do Adulto é uma condição rara caracterizada por febre, artralgia e rash maculopapular que dificilmente caminha com Hipertensão Arterial Pulmonar. Dessa forma, é necessário maior conhecimento sobre a doença pela comunidade médica, com o objetivo de impedir sua progressão e complicações.

Palavras-chaves: Doença de Still do adulto, Hipertensão arterial pulmonar, Reumatologia.

\begin{abstract}
Objective: To report the coexistence between Adult-Onset Still's Disease and Pulmonary Arterial Hypertension. Details of the case: We present the case of a 29-year-old pregnant woman, referred by the rheumatology service of a hospital in the state of São Paulo with a diagnosis of Adult-Onset Still's Disease. She had persistent fever, arthralgia, bilateral wrist arthritis, pericarditis, pleural effusion, elevated ferritin, lymphadenopathy and visceromegaly at the onset of the disease. A cesarean delivery was performed and, two months later, he used progressive doses of prednisolone, after relapse of the disease. She had an evolution of the condition with the same symptoms, but associated with pulmonary hypertension on echocardiographic examination. Transferred to the University of São Paulo for cardiac catheterization. During the examination, she had cardiorespiratory arrest, with no response to resuscitation maneuvers. Final considerations: Adult Still's Disease is a rare condition characterized by fever, arthralgia and maculopapular rash that is difficult to walk with Pulmonary Arterial Hypertension. Thus, greater knowledge about the disease is needed by the medical community, in order to prevent its progression and complications.
\end{abstract}

Keywords: Adult Still's disease, Pulmonary arterial hypertension, Rheumatology.

\section{RESUMEN}

Objetivo: Informar la coexistencia entre enfermedad de Still del adulto e hipertensión arterial pulmonar. Detalles del caso: Presentamos el caso de una mujer embarazada de 29 años, remitida por el servicio de reumatología de un hospital del estado de São Paulo con diagnóstico de Enfermedad de Still del Adulto. Al início de la enfermedad presenta fiebre persistente, artralgias, artritis de muñeca bilateral, pericarditis, derrame pleural, ferritina elevada, adenopatías y visceromegalias. Se realizó cesárea y, dos meses después, utilizó dosis progresivas de prednisolona, tras la recaída de la enfermedad. La evolución del paciente evolucionó con la misma sintomatología, pero se asoció a hipertensión pulmonar en el examen ecocardiográfico. Transferido a la Universidad de São Paulo para cateterismo cardíaco. Durante la exploración presentó parada cardiorrespiratoria, sin respuesta a maniobras de reanimación. Consideraciones finales: La Enfermedad de Still del Adulto es una condición poco común caracterizada por fiebre, artralgia y erupción maculopapular de dificultad para caminar con Hipertensión Arterial Pulmonar. Por tanto, es necesario un mayor conocimiento de la enfermedad por parte de la comunidad médica, para prevenir su progresión y complicaciones.

Palabras clave: Enfermedad de Still del adulto, Hipertensión arterial pulmonar, Reumatología.

${ }^{1}$ Centro Universitário Católico Salesiano Auxilium (UNISALESIANO), Araçatuba - SP.

*E-mail: tccjosemarques@gmail.com

SUBMETIDO EM: 5/2021

ACEITO EM: 6/2021

PUBLICADO EM: 6/2021 


\section{INTRODUÇÃO}

A doença de Still do Adulto (DSA) é um distúrbio inflamatório multissistêmico, sendo caracterizada pela tríade clássica febre persistente, artralgia e rash maculopapular (SCHLESINGER P, 2011; GIACOMELLI R, et al., 2018; FEIST E, et al., 2018; WANG M-Y, et al., 2019). Pouco tempo depois, surgem outros sinais e sintomas, como fadiga, emagrecimento e anorexia. Ela foi descrita pela primeira vez em 1971 por Eric Bywaters como uma condição inflamatória em adultos jovens (BYWATERS EG, 1971). A doença era semelhante a Doença de Still na infância, atualmente conhecida como Artrite Idiopática Juvenil de Início Sistêmico, que foi descrita em 1897 pelo médico George Frederic Still (GIACOMELLI R, et al., 2018).

A febre da DSA é persistente e geralmente se estende por semanas, até que o diagnóstico seja estabelecido. Os picos febris desses pacientes ocorrem diariamente, em geral ao entardecer ou ao anoitecer. Essas elevações da temperatura podem estar associadas a calafrios e sudorese (SCHLESINGER P, 2011; GIACOMELLI R, et al., 2018; FEIST E, et al., 2018; WANG M-Y, et al., 2019).

A erupção da DSA é maculosa, apresenta cor de salmão e pode ocorrer em qualquer região do tronco e dos membros, sendo evanescente e ficando evidente durante os episódios febris, mas desaparece por completo quando a temperatura volta ao normal. As lesões podem ser ligeiramente pruriginosas e estenderse para as áreas escarificadas pelo paciente, caracterizando o fenômeno de Koebner (SCHLESINGER P, 2011; GIACOMELLI R, et al., 2018; FEIST E, et al., 2018; WANG M-Y, et al., 2019).

Dor articular é uma queixa comum na DSA, mas a artrite propriamente dita pode demorar a desenvolverse. Inicialmente, os pacientes costumam ter dores articulares e musculares significativas sem sinovite detectável. Nos estágios iniciais, pode haver artralgias e mialgias acentuadas que, com o transcorrer do tempo, transformam-se em artrite bem desenvolvida. A artrite acomete mais comumente as grandes articulações, como quadris, joelhos, tornozelos, ombros e punhos, em vez das articulações pequenas das mãos e dos pés (SCHLESINGER P, 2011; GIACOMELLI R, et al., 2018; FEIST E, et al., 2018; WANG M-Y, et al., 2019).

Embora apresente várias complicações, existem poucos trabalhos publicados que evidenciam a associação entre Doença de Still do Adulto e Hipertensão Arterial Pulmonar. O objetivo desse estudo é relatar o caso de uma gestante de 29 anos diagnosticada com Doença de Still do Adulto e que evoluiu para quadro de Hipertensão Arterial Pulmonar.

\section{DETALHAMENTO DO CASO}

Este estudo de caso foi aprovado pelo Comitê de Ética em Pesquisa do Centro Universitário Católico Salesiano Auxilium, UniSALESIANO-Araçatuba (CAAE 38505020.6.0000.5379). O Termo de Consentimento Livre e Esclarecido (TCLE) foi obtido através da família da paciente, sendo assinado pelo pai da mesma.

Paciente do sexo feminino, 29 anos, foi referenciada de um hospital do estado de São Paulo para seguimento em Clínica Reumatológica.

Em sua primeira avaliação, abril de 2008, informou gravidez de 6 meses, primigesta, gestação gemelar, sem intercorrências obstétricas ao pré-natal, sem uso de medicação para controle de sua doença e assintomática.

No relatório médico enviado constavam as seguintes informações: paciente com diagnóstico de doença de Still do adulto, que se iniciou há cerca de 1,5 ano, apresentando, progressivamente, desde o início do quadro, oligoartrite simétrica em punhos, febre, pericardite, pleurite, hepatoesplenomegalia e linfoadenopatia. Os exames complementares apresentavam elevação importante de ferritina sérica, leucocitose e auto anticorpos negativos para FAN, anti DNA, anti SM, Anti RNP, anti Ro, anti LA e fator reumatoide.

A enfermidade foi tratada com prednisona $60 \mathrm{mg}$ por dia, com redução lenta e progressiva, até a suspensão do fármaco, com remissão completa clínica e laboratorial. A Paciente informou que estava assintomática desde o início da gravidez, fazendo uso apenas de $5 \mathrm{mg}$ de ácido fólico diariamente. Todos os exames complementares estavam normais ou negativos. 
Após cinco meses retorna à Clínica para reavaliação, informando que foi submetida a parto cirúrgico, sem intercorrências, com nascimento de gêmeos, com sexo masculino e feminino. Queixava-se de episódios de dor leve em punhos e rash cutâneo eventual, mais frequente no período vespertino. Ao exame físico apresentava-se sem artrite, sem dor ao exame dos punhos e sem presença de rash cutâneo. Os exames laboratoriais estavam todos normais ou negativos, considerando-se assim que o quadro continuava em remissão, não sendo introduzido medicação para controle da doença.

Após cerca de seis meses, a paciente apresentou artrite leve em punhos, rash cutâneos eventual, sem febre, com ultrassom abdominal demonstrando pequena hepatoesplenomegalia, com ausência de acometimento pericárdio ou pleural e exames laboratoriais todos normais ou negativos. Em virtude de início de atividade inflamaria da doença foi introduzido prednisona $20 \mathrm{mg}$ por dia e sulfato de hidroxicloroquina 400 mg por dia, ficando assintomática.

Cerca de três meses após apresentou dor de garganta, febre persistente, artrite leve em punhos, poliartralgias, rash cutâneo frequente, verpertino, ao exame físico apresentava fenômeno de Koebner, VHS de $20 \mathrm{~mm}$, PCR $38 \mathrm{mg} / \mathrm{l}$ e ferritina $486 \mathrm{ng} / \mathrm{ml}$. Diante da atividade clínica e laboratorial foi aumentada a dose de prednisona para $40 \mathrm{mg}$ por dia e acrescentado leflunomide $20 \mathrm{mg}$ por dia, com boa resposta clinica no seguimento.

Dois meses após, retorna relatando dor torácica atípica, sem tosse, febre alta persistente, artrite em punhos, rash cutâneo persistente, queixando-se de hipogeusia. Diante do quadro foi realizado pulsoterapia, com uso de metilprednisolona 1 grama por dia, endovenosa, por três dias consecutivos com boa resposta, evoluindo sem artrite, febre ou rash cutâneo.

Decorridos seis meses da última avaliação, a paciente retornou queixando-se de dispneia aos grandes esforços, apresentando edema leve de membros inferiores. O ecocardiograma demonstrou pressão de artéria pulmonar de $70 \mathrm{~mm}$, a tomografia pulmonar não apresentou anormalidade, sendo medicada com metil prednisolona 1 grama endovenosa por dia por três dias e prescrição de sildenafil $25 \mathrm{mg}$ três vezes ao dia, com melhora do quadro de dispneia e edema de membros inferiores.

A paciente foi enviada a um hospital especializado no estado de São Paulo para a realização de cateterismo direito para melhor caracterização da hipertensão da artéria pulmonar. A informação da equipe médica é que na realização do cateterismo direito a paciente apresentou quadro convulsivo, seguido de parada cardiorrespiratória. O Serviço de Verificação de Óbito não definiu a causa do óbito.

\section{DISCUSSÃO}

A Doença de Still do Adulto, moléstia inflamatória sistêmica com sua etiologia a esclarecer, afeta principalmente adultos jovens (GIACOMELLI R, et al., 2018). Descrita em 1971 por Eric Bywaters, em sua série de 14 casos que descrevem pacientes com febre persistente, artrite e rash maculopapular (BYWATERS $E G, 1971)$. Em relação à dados epidemiológicos, sua incidência varia entre 0,16 a $0,4 / 100000$ e sua prevalência entre 1 a 34 casos/ 1 milhão de pessoas. Não há diferenças entre os sexos. Apresenta um pico entre os 15 e 25 anos e também entre os 36 e 46 anos (SCHLESINGER P, 2011; GIACOMELLI R, et al., 2018; FEIST E, et al., 2018; WANG M-Y, et al., 2019).

Diversas explicações são propostas em relação à fisiopatologia da Doença de Still do Adulto, mas é sabido que sua etiopatogenia envolve tanto a imunidade inata quanto a adaptativa. Uma delas propõe a causa pós infecciosa uma vez que diversas infecções virais (EBV, CMV e Hepatites B e C vírus) e bacterianas (Yersinia enterocolitica, Campylobacter jejuni, Chlamydia trachomatis, Chlamydia pneumoniae, Mycoplasma pneumoniae e Borrelia burgdorferi) estão correlacionadas com sua patogenia (GIACOMELLI R, et al., 2018; FEIST E, et al., 2018; GERFAUD-VALENTIN M, et al., 2014).

Em relação ao sistema imune inato, é entendido que o Receptor Toll-like (TLR) 7 tem papel fundamental na promoção e recrutamento de neutrófilos para aumento da resposta inflamatória. Já o sistema imune adaptativo tem como início do processo inflamatório a presença de patógenos (PAMPs) ou de moléculas associadas ao dano tecidual (DAMPs). Esses sinais de perigo são transmitidos aos macrófagos e neutrófilos 
através dos TLRs, que ativam inflamassomas específicos como o NLRP3 (Proteína 3 contendo os domínios NACHT, LRR e PYD), levando a ativação e produção de ILs. Acredita-se que essa fase é essencial para uma intensa produção de várias citocinas pró-inflamatórias, como por exemplo IL-6, IL-8, IL-17, IL-18 e TNF 9,1416, marcando o início da doença (GIACOMELLI R, et al., 2018; FEIST E, et al., 2018; GERFAUD-VALENTIN M, et al., 2014).

Yamaguchi M, et al. (1992) desenvolveram os critérios diagnósticos mais aceitos para Doença de Still do Adulto. Eles são divididos em critérios maiores, menores e de exclusão (Quadro 1). Para a confirmação da doença, é necessário a presença de cinco critérios ou mais, sendo obrigatoriamente a presença de pelo menos dois maiores (YAMAGUCHI M, et al., 1992; GIACOMELLI R, et al., 2018; SINHA A, et al, 2018; WANG $\mathrm{M}-\mathrm{Y}$, et al., 2019). Em relação ao caso apresentado, a paciente apresentava todos os critérios.

Quadro 1 - Critérios de Yamaguchi para diagnóstico de Doença de Still do Adulto.

\begin{tabular}{|c|c|}
\hline I. Critérios Maiores & $\begin{array}{c}\text { Febre }>39^{\circ} \mathrm{C} \text { por pelo menos uma semana; } \\
\text { Artralgia ou artrite por no mínimo duas semanas; } \\
\text { Rash cutâneo; } \\
\begin{array}{c}\text { Leucocitose }> \\
\text { polimorfonucleares. }\end{array}\end{array}$ \\
\hline II. Critérios Menores & $\begin{array}{c}\text { Dor de garganta; } \\
\text { Desenvolvimento recente e significativo de } \\
\text { linfadenopatia; } \\
\text { Hepatomegalia ou Esplenomegalia; } \\
\text { Teste de funções hepáticas alterados; } \\
\text { Teste de anticorpo antinuclear e fator reumatóide } \\
\text { negativos. }\end{array}$ \\
\hline $\begin{array}{l}\text { III. Critérios de } \\
\text { Exclusão }\end{array}$ & $\begin{array}{c}\text { Infecções; } \\
\text { Malignidades; } \\
\text { Outras doenças reumáticas. }\end{array}$ \\
\hline
\end{tabular}

Fonte: Garbin AJS, et al., 2021; adaptado de Yamaguchi M, et al., 1992.

A Hipertensão Arterial Pulmonar (HAP) é definida como o aumento dos níveis pressóricos na circulação pulmonar devido ao aumento da resistência vascular. Ela pode ocorrer tanto por doenças de causa pulmonares ou extrapulmonares. A HAP é uma condição determinada pela pressão arterial pulmonar média igual ou acima de $25 \mathrm{mmHg}$ em repouso (PRADA LFL, et al., 2014; MARON BA e GALIÈ N, 2016; THENAPPAN T, et al., 2018).

Por conta de sua importância e variedade clínica, a HAP passou por uma série de classificações ao longo dos anos com o objetivo de agrupar suas mais variadas formas, baseando-se desde sua etiopatogenia até suas condutas terapêuticas (PRADA LFL, et al., 2014).

De acordo com o V Simpósio Mundial de Hipertensão Pulmonar, a HAP é dividida em cinco subgrupos (PRADA LFL, et al., 2014; MARON BA e GALIÈ N, 2016; THENAPPAN T, et al., 2018). Cada um deles recebe uma denominação e reúne uma série de condições relacionada ao nome na qual pertence (Quadro 2). 
Quadro 2 - Subgrupos da Hipertensão Arterial Pulmonar.

\begin{tabular}{|c|c|}
\hline I. Hipertensão Arterial Pulmonar & $\begin{array}{l}\text { 1.1. Idiopática } \\
\text { 1.2. Hereditária } \\
\text { 1.3. Induzida por Drogas } \\
\text { 1.4. Associada a: } \\
\text { 1.4.1. Doenças do tecido conjuntivo } \\
\text { 1.4.2. Infecção por HIV } \\
\text { 1.4.3. Hipertensão Portal } \\
\text { 1.4.4. Doenças Cardíacas Congênitas } \\
\text { 1.4.5. Esquistossomose } \\
\text { 1' Doença veno-oclusiva pulmonar e/ou hemangiomatose capilar } \\
\text { pulmonar } \\
\text { 1" Hipertensão Pulmonar persistente do recém-nascido }\end{array}$ \\
\hline $\begin{array}{l}\text { II. Hipertensão Pulmonar por Doença } \\
\text { Cardíaca Esquerda }\end{array}$ & $\begin{array}{l}\text { 1.1. Disfunção sistólica do ventrículo esquerdo } \\
\text { 1.2. Disfunção diastólica do ventrículo esquerdo } \\
\text { 1.3. Doença valvular } \\
\text { 1.4. Obstrução congênita/adquirida da via de saída do ventrículo } \\
\text { esquerdo e miocardiopatias congênitas } \\
\text { 1.5. Estenose congênita/adquirida de veias pulmonares }\end{array}$ \\
\hline $\begin{array}{l}\text { III. Hipertensão Pulmonar devido à } \\
\text { Doença Pulmonar ou Hipoxemia }\end{array}$ & $\begin{array}{l}\text { 1.1. Doença Pulmonar Obstrutiva Crônica } \\
\text { 1.2. Doença Intersticial Pulmonar } \\
\text { 1.3. Outras doenças pulmonares com padrão misto obstrutivo e } \\
\text { restritivo } \\
\text { 1.4. Doença respiratória do sono } \\
\text { 1.5. Hipoventilação alveolar } \\
\text { 1.6. Exposição crônica a alta altitude } \\
\text { 1.7. Anomalias do desenvolvimento do pulmão }\end{array}$ \\
\hline $\begin{array}{l}\text { IV. Hipertensão Pulmonar por } \\
\text { doença tromboembólica crônica e } \\
\text { outras obstruções da artéria } \\
\text { pulmonar }\end{array}$ & $\begin{array}{l}\text { 1.1. Hipertensão Pulmonar tromboembólica crônica } \\
\text { 1.2. Outras obstruções da artéria pulmonar } \\
\text { 1.2.1. Angiossarcoma } \\
\text { 1.2.2. Outros tumores intravasculares } \\
\text { 1.2.3. Arterites } \\
\text { 1.2.4. Estenose congênita da artéria pulmonar } \\
\text { 1.2.5. Parasitas (Hidatidose) }\end{array}$ \\
\hline $\begin{array}{l}\text { V. Hipertensão Pulmonar por } \\
\text { Mecanismo Multifatorial } \\
\text { Desconhecido }\end{array}$ & $\begin{array}{l}\text { 1.1. Doenças hematológicas: anemia hemolítica crônica, } \\
\text { síndromes mieloproliferativas, esplenectomia } \\
\text { 1.2. Doenças sistêmicas, sarcoidose, histiocitose pulmonar de } \\
\text { células de Langherans, linfangioleiomiomatose } \\
\text { 1.3. Doenças metabólicas: doença do armazenamento do } \\
\text { glicogênio, doença de Gaucher, tireoidopatias } \\
\text { 1.4. Outras: obstrução tumoral, mediastinite fibrosante, } \\
\text { insuficiência renal crônica, hipertensão pulmonar segmentar }\end{array}$ \\
\hline
\end{tabular}

Fonte: Garbin AJS, et al., 2021; adaptado de Prada LFL, et al., 2014; Maron BA e Galiè N, 2016; Thenappan T, et al., 2018. 
Primeiramente, a HAP é assintomática em pacientes com doenças reumáticas autoimunes (SHAHANE A, 2013; SEGURO LPC e SOUZA R, 2014). Com o avanço da enfermidade, os pacientes podem apresentar hiperfonese de $2^{\circ}$ bulha em foco pulmonar, sopros de insuficiência pulmonar ou tricúspide e sinais de insuficiência cardíaca direita com edema de membros inferiores, refluxo hepatojugular e presença de galope do ventrículo direito (SHAHANE A, 2013; SEGURO LPC e SOUZA R, 2014; SALVADOR MJ, 2014; GALIĖ N, et al., 2015). Outros sintomas relatados são fadiga, dor precordial, dispneia aos esforços, tontura e síncope (SHAHANE A, 2013; SEGURO LPC e SOUZA R, 2014; GALIÈ N, et al., 2015). Em relação ao caso, a paciente apresentava alguns desses sintomas e também queixa de hipogeusia, porém a sua provável etiologia foi 0 uso de leflunomida (FILHO JM, 2016).

O ecocardiograma é o principal exame solicitado para triagem da HAP sendo utilizados três parâmetros hemodinâmicos para a avaliação do quadro: Pressão Sistólica da Artéria Pulmonar (PSAP), Velocidade de Regurgitação Tricúspide (VRT) e a Excursão Sistólica do Plano Anular Tricúspide (TAPS). Além disso, existem outros achados sugestivos de hipertensão pulmonar, como disfunção sistólica do ventrículo direito, aumento do átrio direito e regurgitação tricúspide (SHAHANE A, 2013; SEGURO LPC e SOUZA R, 2014; SALVADOR MJ, 2014; GALIÈ N, et al., 2015).

Dentre as doenças reumáticas autoimunes, a HAP é mais frequente na esclerose sistêmica, acometendo cerca de $12 \%$ dos pacientes e sendo sua principal causa de morte. Por conta disso, o ecocardiograma deve ser realizado periodicamente nos pacientes com essa enfermidade. O diagnóstico definitivo da hipertensão pulmonar é firmado com o cateterismo cardíaco direito, sendo realizada a medida direta das pressões das câmaras cardíacas direitas e da artéria pulmonar (SHAHANE A, 2013; SEGURO LPC e SOUZA R, 2014; SALVADOR MJ, 2014; GALIÈ N, et al., 2015).

Foram descritos sete casos que relacionam Doença de Still do Adulto e Hipertensão Arterial Pulmonar (ZEN A, et al., 1990; MUBASHIR E, et al., 2006; SIQUEIRA MEM, et al, 2009; CAMPOS M e SCHIOPU E, 2012; KADAVATH S, et al, 2014; GUILLERMINAULT L, et al, 2016; SINHA A, et al, 2018).

Embora a literatura evidencie que não há preferência entre os sexos, foram observados seis casos em mulheres (ZEN A, et al., 1990; MUBASHIR E, et al., 2006; CAMPOS M e SCHIOPU E, 2012; KADAVATH S, et al, 2014; GUILLERMINAULT L, et al, 2016; SINHA A, et al, 2018).

A faixa etária preferencialmente acometida encontra-se entre os 21 a 30 anos (ZEN A, et al., 1990; MUBASHIR E, et al., 2006; CAMPOS M e SCHIOPU E, 2012; KADAVATH S, et al, 2014; GUILLERMINAULT L, et al, 2016; SINHA A, et al, 2018).

Observa-se que a HAP pode se apresentar precoce ou tardiamente no curso da doença. De acordo com a classificação mundial da Hipertensão Arterial Pulmonar, a HAP associada à Doença de Still enquadra-se no grupo 1, o qual encontram-se as condições idiopáticas, hereditárias, induzida por drogas e doenças do tecido conjuntivo (SHAHANE A, 2013; SEGURO LPC e SOUZA R, 2014; SALVADOR MJ, 2014; GALIĖ N, et al., 2015). Embora seja considerada uma condição grave, dos sete pacientes relatados, apenas dois evoluíram à óbito (MUBASHIR E, et al., 2006; GUILLERMINAULT L, et al, 2016).

Yamaguchi M, et al. (1992) afirmam que a DSA é uma doença inflamatória multissistêmica cujo diagnóstico se baseia em critérios clínicos e laboratoriais. A HAP faz parte das manifestações de vasculopatia associada a doenças reumáticas autoimunes, principalmente na esclerose sistêmica (SHAHANE A, 2013; SEGURO LPC e SOUZA R, 2014; SALVADOR MJ, 2014; GALIÈ N, et al., 2015). O ecocardiograma é um exame fundamental para sua triagem, sendo o cateterismo cardíaco o padrão-ouro para seu diagnóstico (SHAHANE A, 2013; SEGURO LPC e SOUZA R, 2014; SALVADOR MJ, 2014; GALIÈ N, et al., 2015).

Portanto, após análise do trabalho, concluímos que a Doença de Still do Adulto é uma condição rara sendo pouco relatado na literatura mundial a sua coexistência com Hipertensão Arterial Pulmonar. Esse estudo de caso apresentou contribuições científicas, pois além de apresentar um caso de raridade, evidencia que a associação dessas enfermidades é grave e pode levar o paciente à óbito. Por conta disso, é essencial que os profissionais da saúde tenham esse conhecimento para que possam guiar da melhor forma possível casos similares em suas carreiras. 


\section{REFERÊNCIAS}

1. BYWATERS E G. Still's disease in the adult. Ann Rheum Dis 1971; 30: 121-133.

2. CAMPOS M, SCHIOUPI E. Pulmonary Arterial Hypertension in Adult-Onset Still's Disease: rapid response to anakinra. Case Reports In Rheumatology 2012; 2012: 1-5.

3. FEIST E, et al. Mechanisms, biomarkers and targets for adult-onset Still's disease. Nature Reviews Rheumatology Publish Online First: 14 September 2018. doi: 10.1038/s41584-018-0081-x.

4. FILHO JM. Hypogeusia as a side effect of leflunomide. ACTA Reumatol Port 2016; 41: 88-89.

5. GALIÈ N, et al. Guidelines for the diagnosis and treatment of pulmonary hypertension. Eur Heart J Online First 29 August 2015. doi: 10.1093/eurheartj/ehv317.

6. GERFAUD-VALENTIN M, et al. Adult-onset Still's disease. Autoimmunity Reviews Publish Online First: 19 March 2014. doi: 10.1016/j.autrev.2014.01.058.

7. GIACOMELLI R, et al. A comprehensive review on adult onset Still's disease. Journal of Autoimmunity 2018; 93: 2436.

8. GUILLEMINAULT L, et al. Pulmonary arterial hypertension in adult onset Still's disease: a case report of a severe complication. BMC Pulmonary Medicine Publish Online First: 10 May 2016. doi:10.1186/s12890-016-0237-x.

9. KADAVATH S, et al. A novel therapeutic approach in pulmonary arterial hypertension as a complication of adult-onset Still's disease: targeting il-6. International Journal Of Rheumatic Diseases 2014; 17: 336-340.

10. MARON BA, GALIĖ N. Diagnosis, Treatment, and Clinical Management of Pulmonary Arterial Hypertension in the Contemporary Era. Jama Cardiology Publish Online First: 16 November 2016. doi: 10.1001/jamacardio.2016.4471.

11. MUBASHIR E, et al. Pulmonary hypertension in a patient with adult-onset stills disease. Clinical Rheumatology Publish Online First: 27 July 2006. doi:10.1007/s10067-006-0382-3.

12. PRADA LFL, et al. Classificação da hipertensão pulmonar. Rev Paul Reumatol 2014; 13(2): 6-15.

13. SALVADOR MJ. Hipertensão Pulmonar - diagnóstico, referenciação, novos avanços terapêuticos. ACTA Reumatol Port 2014; 39: 16-17.

14. SCHLESINGER P. Adult-Onset Still's Disease. In: Imboden J, Hellmann D, Stone J. CURRENT Diagnosis and Treatment in Rheumatology. New York: McGraw Hill, 2011: 193-195.

15. SEGURO LPC, SOUZA R. Investigação diagnóstica da hipertensão pulmonar. Rev Paul Reumatol 2014; 13(2): $17-23$.

16. SHAHANE A. Pulmonary hypertension in rheumatic diseases: epidemiology and pathogenesis. Rheumatol Int Online First 19 January 2013. doi: 10.1007/s00296-012-2659-y.

17. SINHA A, et al. Severe Pulmonary Hypertension Due to Adult-Onset Still's Disease. Journal Of Investigative Medicine High Impact Case Reports 2018; 6: 1-4.

18. SIQUEIRA MEM, et al. Adult-onset Still's disease and pulmonary arterial hypertension. Respiratory Medicine CME 2009; $2:$ 70-72.

19. THENAPPAN T, et al. Pulmonary arterial hypertension: pathogenesis and clinical management. The BMJ Publish Online First: 14 March 2018. doi: 10.1136/bmj.j5492.

20. WANG W-Y, et al. Pathogenesis, disease course, and prognosis of adult-onset Still's disease: an update and review. Chinese Medical Journal 2019; 132(23): 2856-2864.

21. YAMAGUCHI M, et al. Preliminary criteria for classification of adult Still's disease. J Rheumatol.1992; 19: $424-430$.

22. ZEN A, et al. A case of adult Still's disease with pulmonary hypertension. Ryumachi 1990; 30: 45-52. 